\title{
Editorial
}

\section{Making Engineering Accessible to Medics}

Research and development in bioengineering and medical technology, conducted during recent decades, have led to spectacular progress in clinical medicine. These achievements have triggered a huge increase in the number of courses offered in the areas of bioengineering, clinical technology and medical informatics; nowadays, most major universities offer curricula oriented towards these fields. The majority of participants however come from engineering backgrounds and so modules dealing with basic biological and medical sciences have been included. These have been facilitated by the ready availability of textbooks in this area, many of which were specifically written for nursing A \& P (Anatomy and Physiology) programmes.

In contrast, relatively few participants from medicine have taken up courses in biomedical engineering, to the detriment of scientific exchanges between engineers and medical doctors. The reasons for this imbalance are many and may vary from country to country, but a consistent finding is the difficulty (or courage) in taking the first step. 'Introductory' texts in bioengineering tend to involve vector algebra and calculus early in Chapter 1. While in most countries entry to medical school is very competitive and requires, among others, high grades in mathematics, little more than arithmetic is required during the course itself, so numeracy undergoes disuse atrophy.

Furthermore, to paraphrase George Bernard Shaw, medics and engineers are separated by a common language. To the medic, stress and strain are both symptoms of anxiety, while to the engineer they are defined by equations involving symbols such as $\sigma$ and $\varepsilon$ and are related to forces and deformations. To the average medic this is, literally, all Greek.

ESEM is a bridge between medicine and engineering. It promotes cultural and scientific exchanges between engineers and medics and training courses in biomedical engineering. What better way to achieve these objectives than to address this problem and help medics to take this first step?

To this end, we are beginning a new series of First Step papers in Technology \& Health Care aimed at medics, to help bring them to the level where they can begin an MSc in biomedical engineering, or at medics who "simply" wish to understand a particular medical technology. Written by engineers who are leaders in their field, with input from medical colleagues, they will cover the basic engineering principles underpinning biomechanics, bioelectronics, medical informatics, biomaterials, tissue engineering, bioimaging and rehabilitation engineering, and will include clinically relevant examples.

As Albert Einstein may have said 'Everything should be made as simple as possible, but not simpler'.

Clive Lee and Peter Niederer 